\title{
Model study of multiple polar cap arcs: Occurrence and spacing
}

\author{
L. Zhu, J. J. Sojka, R. W. Schunk, and D. J. Crain \\ Center for Atmospheric and Space Science, Utah State University, Logan, Utah
}

Abstract. A new scenario for the formation of multiple polar cap arcs is proposed based on the results from a timedependent electrodynamic model of polar cap arcs developed by Zhu et al. [1993]. The results suggest that the appearance by multiple polar cap arcs may not be due to multiple structures in the magnetospheric source region, but instead, may in rimarily be determined by the coupled magnetosphereonosphere system in which the ionosphere plays an active role. It was found that with the same magnetospheric driver, a strong ionospheric background convection and an ionospheric background Hall conductance in the range of from 0.5 to 1.5 mho favor the occurrence of multiple polar cap arcs. It was also found that the spacing between individual arcs is highly correlated with the ratio of the enhanced Hall and Pedersen conductances (i.e., the hardness of the primary magnetospheric precipitation), which is allowed to vary in the model runs. A harder precipitation causes a wider spacing. In the modelings, the spacing ranged from about 20 kilometers to about 60 kilometers when the precipitation hardness increased.

\section{Introduction}

The polar cap arcs are the auroral arcs seen at very high geomagnetic latitudes $\left(>80^{\circ}\right)$ that extend approximately $10 \sim$ 15 degree in the sun-aligned direction. Some of these arcs are very bright and may extend across the polar cap from the dayside to the nightside of the auroral oval to form a pattern that resembles the Greek letter "theta" when observed from space [Frank et al., 1986]. However, polar cap arcs can be confined in the polar cap and be relatively weak, can have multiple discrete structures, or can occur in either the evening or morning sectors of the polar cap [Weber and Buchau, 1981].

Compared to the observations, the theoretical understanding of polar cap arcs is still in its infancy. Most of the theoretical models of polar cap arcs [e.g., Burke et al., 1982] are either qualitative, semiquantitative, or steady state models. In these theoretical studies, the emphasis was on the magnetospheric role and the active role of the ionosphere in the magnetosphere-ionosphere (M-I) coupling processes of polar cap arcs was ignored. The main concerns were on the bright transpolar arcs, with little attention paid to the relatively weak polar cap arcs that exhibit multiple structures.

Zhu et al. [1993] developed a time-dependent model of polar cap arcs in which the electrodynamics of the polar cap arcs is treated self-consistently in the frame of the coupled M-I system and the active role of the ionosphere is specifically stressed. Based on their modeling results, Zhu et al. [1993] proposed that the observed multiple polar cap arcs might not be due to multiple structures in the magnetospheric source region. Instead, they suggested that such striations naturally occur as a result of the dynamical processes that operate in the coupled M-I system, with the ionosphere playing a key role in the formation of multiple polar cap arcs. In a subsequent study, Sojka et al. [1993] found a strong dependence of the occurrence of multiple polar cap arcs on the large-scale ionospheric background convection. These studies have provided a new insight for the formation of polar cap arcs and revealed the active role of the ionosphere in the dynamics of

Copyright 1994 by the American Geophysical Union.

Paper number 94GL00562

0094-8534/94/94GL-00562\$03.00 polar cap arcs. The work presented in this paper is a follow-on to the Zhu et al. [1993] and Sojka et al. [1993] studies. The goal of the paper is to provide a more complete theoretical picture of the formation of multiple polar cap arcs by combining the key results from these previous studies and the results from the new model simulations into an integrated theoretical picture. One of the major features in the new model simulations is that the ratio of the Hall and Pedersen conductances is a variable, instead of a constant as in the previous work.

\section{Theoretical Model}

The key aspect in the model of polar cap arcs developed by Zhu et al. [1993] is that the arcs are not treated as phenomena merely controlled by the magnetosphere, with the ionosphere only acting as a passive load. Instead, the polar cap arcs are treated as features which are coherently determined by the dynamics in both the ionosphere and magnetosphere. Our scenario for simulating polar cap arcs is briefly summarized as follows. Initially, a magnetospheric shear flow carried by Alfvén waves propagates towards the ionosphere. The significance of the assumed initial magnetospheric shear flow in the model has been discussed by Zhu et al. [1993] and is not repeated here. The downward propagating Alfvén waves are partially reflected from the ionosphere, and then bounce back and forth between the ionosphere and magnetosphere. The nature of the wave reflections depends on both the conditions in the ionosphere and magnetosphere. At the present stage of the model, the wave reflection at the magnetospheric side is simplified by using a reflection coefficient which can vary from -1 to +1 , depending on the magnetic configuration in the magnetospheric source regions. In this work the reflection coefficient is -1 everywhere in the calculation domain, representing an open magnetopause. The propagating Alfvén waves carry both upward and downward field-aligned currents. The precipitating electrons associated with upward fieldaligned currents enhance the conductivity in the ionosphere. The modified ionospheric conductivity launches secondary Alfvén waves towards the magnetosphere. The upward propagating Alfvén waves, which consist of the reflected waves and the secondary Alfvén waves launched by the temporal change of the ionospheric conductivity, carry the ionospheric information back to the magnetosphere, thus reflecting the active ionospheric role in the dynamics of the $\mathrm{M}$-I coupling process. The whole process is transient, during which all physical quantities in the ionosphere change selfconsistently in time, and subsequently, polar cap arcs develop. Due to the finite conductivity in the ionosphere, the temporal variation of the Alfven waves in the coupled M-I system diminishes with time, and the M-I system, as well as the development of polar cap arcs, approach an asymptotic steady state after several bounce periods.

The main initial inputs to the model include: the ionospheric background conductivity defined by the solar and seasonal conditions; the large-scale ionospheric background convection; and the small-scale magnetospheric shear flow associated with polar cap arcs. For the details of the model formulation and calculation procedure, the reader is referred to Zhu et al. [1993].

\section{Occurrence of Multiple Polar Cap Arcs}

By using a shear flow of magnetospheric origin, which extends uniformly along the sun-aligned direction ( $\mathrm{x}$ direction) and has a "single" precipitation channel, as the initial driver 
for the model, Zhu et al. [1993] found that the simulated polar cap arcs can have "multiple" structures in the asymptotic state. The top panel in Figure 1 shows the dawn-dusk (y direction) profile of the field-aligned current distribution associated with the initial shear flow carried by a downward propagating Alfvén wave. This field-aligned current distribution has an upward field-aligned current in the center and downward fieldaligned currents at the two edges. If the ionosphere is just a passive load, as assumed in most of the previous theoretical models of polar cap arcs, a single arc is expected to appear in the ionosphere, since the precipitating electrons associated with the upward current can only produce ionization and emission with a single peak in the center. When the active role of the ionosphere in the M-I coupling processes is included, the features of the simulated polar cap arcs can be different. The remaining panels in Figure 1 show the dawndusk distributions of the field-aligned current, enhanced Hall conductance, and Joule heating rate at the asymptotic state. The negative value in the field-aligned current profile means an upward field-aligned current. Obviously, from the two upward current channels we would infer two polar cap arcs. The spacing of the arcs (the width of the downward current region between the two upward current sheets) in this case is around 50 kilometers, which is different than the characteristic spatial scale of the initial shear flow (about 200 kilometers). Zhu et al. [1993] also found from their simulations that in the development of multiple polar cap arcs, a single arc is seen first and this arc is directly associated with the initial magnetospheric precipitation (we call it the primary arc). After a few minutes, a second arc appears at a distance of tens of kilometers from the first one (we call it a secondary arc), which results from the M-I coupling processes.

The next question is what are the key parameters controlling the occurrence of multiple polar cap arcs in the M-I system. By using the model developed by Zhu et al. [1993], Sojka et al. [1993] found that the appearance of multiple polar cap arcs has a strong dependence on the magnitude of the large-scale ionospheric background convection (E). With the same initial magnetospheric driver, they found that the number of the arcs increased with an increase in the strength of the ionospheric background convection, while the spacing between the arcs remained constant. The degree of striation, or multiple
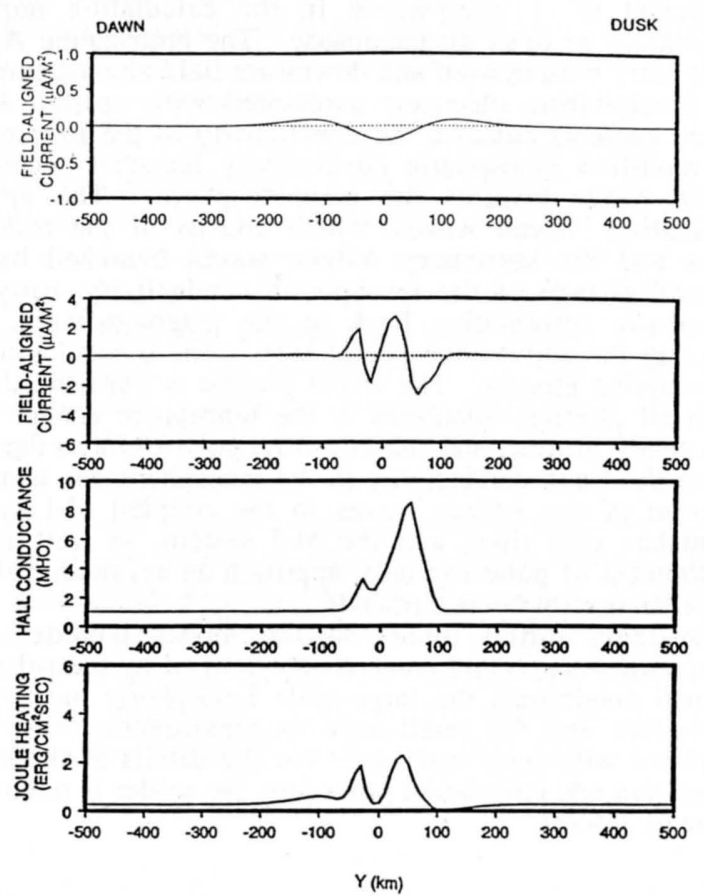

Figure 1. Field-aligned current distribution associated with the initial magnetospheric shear flow (top) and the asymptotic distributions of the field-aligned currents (second), Hall conductance (third), and Joule heating rate (bottom) along the dawn-dusk cross-section.

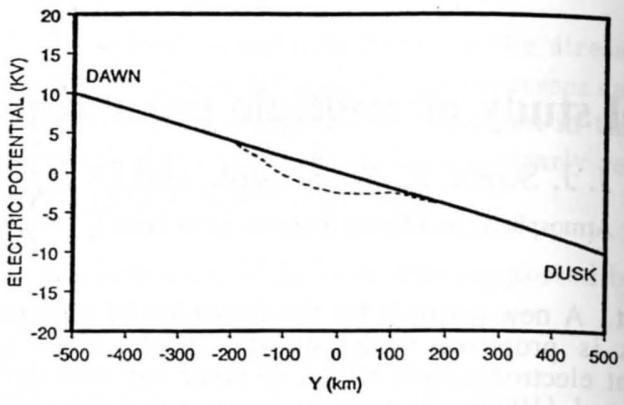

INITLL PERTURBATION

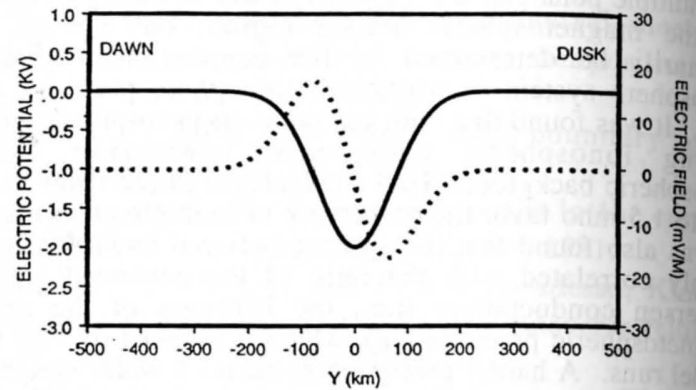

Figure 2. Main initial inputs for the model runs. The top panel shows the ionospheric background potential distribution in the dawn-dusk cross-section (solid curve) and the potential perturbation associated with the initial magnetospheric shear flow (dashed curve). The bottom panel shows the potential distribution associated with the initial magnetospheric shear flow (solid curve) and the corresponding electric field distribution (dashed curve).

character, of the arcs increases rapidly from a single arc at $\mathrm{E}<$ $20 \mathrm{mV} / \mathrm{m}$ to 5 arcs at $30 \mathrm{mV} / \mathrm{m}$. These results further indicate that the ionosphere plays an active role in the formation of multiple polar cap arcs and that the ionosphere dynamically responds to the magnetospheric driving.

From our new simulations, we also found that the ionospheric background "conductivity" influences the occurrence of multiple polar cap arcs. Figure 2 shows the initial inputs adopted for the simulations. The large-scale ionospheric convection shown in the top panel represents a uniform antisunward convection. The initial magnetospheric shear flow carried by a downward propagating Alfvén wave is represented by a Gaussian potential distribution, as shown by the solid line in the bottom panel of Figure 2 . The dashed line represents the corresponding electric field.

Figure 3 shows the asymptotic field-aligned current distribution for the case when the ionospheric background

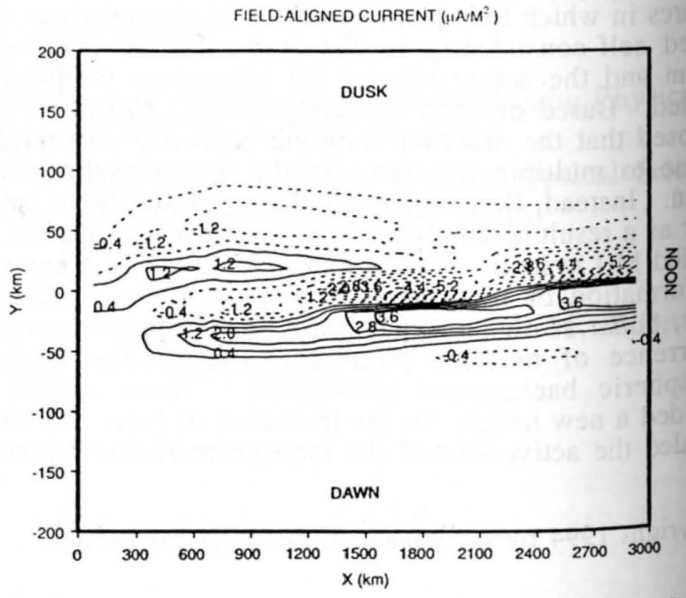

Figure 3. Asymptotic field-aligned current distribution showing the occurrence of multiple polar cap arcs due to the effect of the background ionospheric conductance. 
conductance, which is merely due to solar radiation, is uniform in the dawn-dusk direction and decreases from 2.5 mho on the dayside to 0.5 mho on the nightside. It can be seen that in the regions where the ionospheric background Hall conductance is above 1.5 mho, only a single bright polar cap arc exists. This can be explained by the fact that the high ionospheric conductance allows the magnetospheric current to be freely closed in the ionosphere, which acts to smooth localized discrete structures. In the regions where the ionospheric Hall conductance is in the range of from 0.5 to $1.5 \mathrm{mho}$, there are multiple polar cap arcs. A simulation for an extreme situation showed that when the ionospheric Hall conductance is below $0.5 \mathrm{mho}$, no arcs form in the polar cap. This is because the ionosphere has a very large resistivity and the magnetospheric currents cannot close in the ionosphere; hence, the current flow is cut off in the M-I circuit. These results may explain the observed variety of polar cap arcs, including single bright arcs, multiple arcs, and single arcs with bifurcation at one end. The change of the ionospheric background conductance in the polar cap is mainly due to the variation of solar EUV and UV radiations. The details of how the features of multiple polar cap arcs depend on the solar and seasonal variations will not be discussed in this short paper.

\section{Spacing of Multiple Polar Cap Arcs}

Based on the image data from an all-sky camera, Valladares (private communication, 1993) found that the spatial separation between the arcs can range from tens of kilometers to over one hundred kilometers. In the preceding section, we indicated that the characteristic spatial scale of the spacing of multiple polar cap arcs is different from the characteristic spatial scale of the initial magnetospheric driver. We also noted that the ionospheric background convection and the ionospheric background conductance only influence the occurrence of the multiple polar cap arcs, not their spacing. Therefore, other parameters in the M-I system must determine the spacing of multiple polar cap arcs.

To explore this specific aspect of multiple polar cap arcs, we have made a systematic parametric study using the model developed by Zhu et al. [1993]. We found that the characteristic spatial scale of the initial magnetospheric shear flow does not influence the spacing of multiple polar cap arcs. It only affects the width of the primary arc associated with the initial precipitation. However, we did find an ionospheric parameter that the spacing of the multiple polar cap arcs is sensitive to, that is, the ratio of the Hall and Pedersen conductances, which is an adjustable parameter in the model.

It is commonly known that the Hall-to-Pedersenconductance ratio $\left(\Sigma_{H} / \Sigma_{P}\right)$ is an indicator of the hardness of the electron precipitation. A higher conductance ratio reflects a harder electron precipitation. In our new simulations, we varied the conductance ratio while keeping the other parameters unchanged to see the effect of the hardness of the precipitation on the multiple polar cap arcs. The rationale for these parametric runs is based on the observational fact that for a given field-aligned current the hardness of the precipitation can vary markedly. The variation of the relationship of the field-aligned current and the hardness of the associated precipitation should be largely determined by the dynamical processes in the magnetospheric source regions. The ionospheric background convection and the initial shear flow used in the simulations were the same as those shown in Figure 2. The ionospheric background Hall conductance for the new simulations was uniform in the dawn-dusk direction and decreased from 1.5 mho on the dayside to 0.5 mho on the nightside. The ratio of the "background" Hall and Pedersen conductances was a constant (1.5) for all runs, but the ratio of the "enhanced" Hall and Pedersen conductances caused by the arc precipitation varied from 1 to 2 .

Figure 4 shows the field-aligned current distributions of the asymptotic polar cap arcs for various conductance ratios. The spacing of the multiple polar arcs in this paper is defined as the edge-to-edge distance between two arcs, or the thickness of the downward current sheet embedded between two upward current sheets. It can be seen from Figure 4 that when the
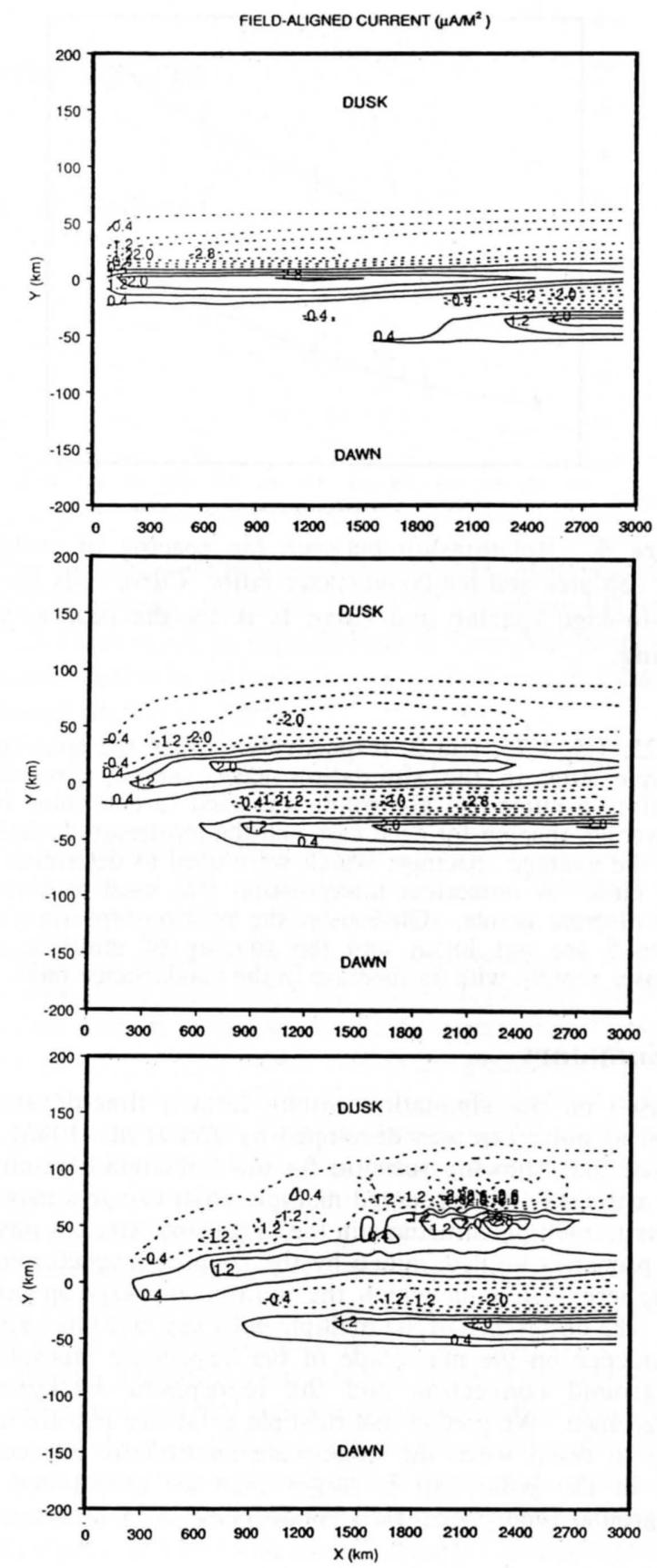

Figure 4. The asymptotic 2-D distributions of the fieldaligned currents for ionospheric conductance ratios $\Sigma_{H} / \Sigma_{P}=1$ (top), 1.5 (middle), and 2 (bottom).

conductance ratio changes from 1 to 2 , the spacing between the primary and secondary arcs increases from about 20 kilometers to about 60 kilometers. This means that a harder precipitation (larger conductance ratio) causes a wider spacing between the primary and secondary arcs, and vice versa. This is an observable theoretical prediction and it can be tested by combining satellite particle precipitation data and groundbased all-sky camera data.

To further elucidate the above theoretical predi ion, we show the quantitative relationship between the conductance ratio and the spacing of multiple arcs in Figure 5. The peak-topeak spacing of multiple arcs is defined as the spatial separation of the peak brightness (or maximum field-aligned current intensity) of two arcs. The relationship shown in Figure 5 is based on the results from five case runs using the polar cap arc model, in which the conductance ratio was set to 


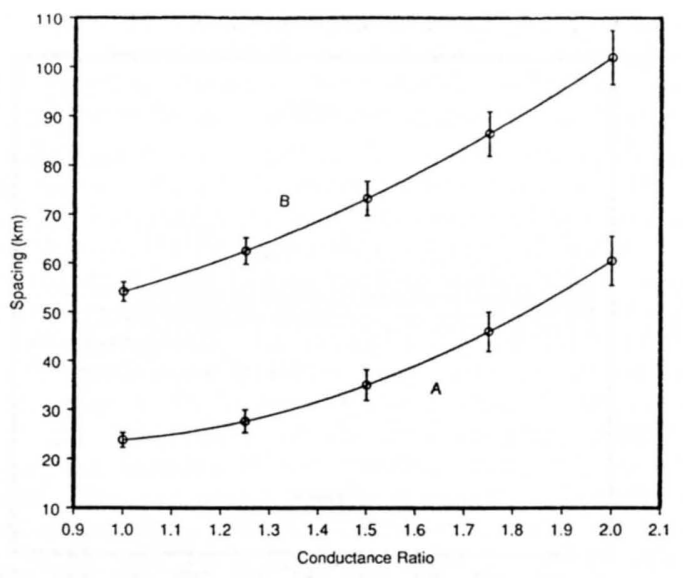

Figure 5. Relationship between the spacing of multiple polar cap arcs and the conductance ratio. Curve $\mathrm{A}$ is for the edge-to-edge spacing and curve $\mathrm{B}$ is for the peak-to-peak spacing.

$1,1.25,1.5,1.75$, and 2 , respectively. Since the spacing of multiple arcs in the simulation has a small noon-night variation, a numerical algorithm was used to determine both the average spacing for each case and the maximum deviations from the average spacings, which were used to determine the error bars. A numerical interpolation was used to connect these discrete points. Obviously, the relationships shown in Figure 5 are not linear and the spacing of multiple arcs increases rapidly with an increase in the conductance ratio.

\section{Summary}

Based on the simulation results from a time-dependent model of polar cap arcs developed by Zhu et al. [1993], we propose the following scenario for the formation of multiple polar cap arcs. The observed multiple polar cap arcs may not be due to multiple structures in the magnetosphere, but instead may primarily be determined by the coupled magnetosphereionosphere system in which the ionosphere plays an active role. The occurrence of the multiple polar cap arcs has a strong dependence on the magnitude of the large-scale ionospheric background convection and the ionospheric background conductance. We predict that multiple polar cap arcs are more likely to occur when the large-scale ionospheric convection field in the polar cap is larger than $20 \mathrm{mV} / \mathrm{m}$ and the background ionospheric Hall conductance is in the range of from 0.5 to 1.5 mho. When the large-scale convection is weak and the ionospheric conductance is high, a single arc is more likely to be seen in the polar cap. With a variable conductance ratio, it was found that the spacing of multiple polar cap arcs is mainly determined by the hardness of the primary magnetospheric precipitation. A harder precipitation causes a wider spacing between individual arcs, and vice versa. The edge-to-edge spacing between arcs varies from about $20 \mathrm{~km}$ to about $60 \mathrm{~km}$ when the ratio of the enhanced Hall and Pedersen conductances changes from 1 to 2 .

It should be noted that the above theoretical predictions are only based on numerical simulations, and they need further physical explanation and a detailed experimental verification. A follow-up study along this line is being undertaken, which consists of both theory and model-observation comparisons. The latter is a component of the NSF/CEDAR(Coupling Energetics and Dynamics of Atmospheric Regions)/HLPS(High Latitude Plasma Structures) campaign in which ground-based optical image data and DMSP satellite data will be used for the model-observation comparisons and model validations.

Acknowledgments. This research was supported by NASA grant NAG5-1484 and by NSF grants ATM-93-08163 and ATM-93-02165 to Utah State University.

\section{REFERENCES}

Burke, W. J., M. S. Gussenhoven, M. C. Kelley, D. A. Hardy, and F. J. Rich, Electric and magnetic characteristics of discrete arcs in the polar cap, J. Geophys. Res., 87, 2431, 1982.

Frank, L. A., J. D. Craven, D. A. Gurnett, S. D. Shawhan, D. R. Weimer, J. L. Burch, J. D. Winningham, C. R. Chappell, J. H. Waite, R. A. Heelis, N. C. Maynard, M. Sugiura, W. K. Peterson, and E. G. Shelley, The theta aurora, J. Geophys. Res., 91, 3177, 1986.

Sojka, J. J., L. Zhu, D. J. Crain, and R. W. Schunk, Effect of high latitude ionospheric convection on sun-aligned polar cap arcs, J. Geophys. Res., in press, 1993.

Weber, E. J., and J. Buchau, Polar cap F-layer auroras, Geophys. Res. Lett., 8, 125, 1981.

Zhu., L., J. J. Sojka, R. W. Schunk, and D. J. Crain, A time-dependent model of polar cap arcs, J. Geophys. Res., 98, 6139, 1993.

D. J. Crain, R. W. Schunk, J. J. Sojka, and L. Zhu, Center for Atmospheric and Space Sciences, Utah State University, Logan, Utah 84322-4405 (e-mail: zhu@cc.usu.edu)

(Received: November 5, 1993; revised: January 19, 1994; accepted: February 9, 1994) 